\title{
Geometry of meandering and braided gravel-bed threads from the Bayanbulak Grassland, Tianshan, P. R. China
}

\author{
François Métivier $^{1}$, Olivier Devauchelle ${ }^{1}$, Hugo Chauvet ${ }^{1}$, Eric Lajeunesse $^{1}$, Patrick Meunier ${ }^{2}$, \\ Koen Blanckaert ${ }^{3}$, Peter Ashmore ${ }^{4}$, Zhi Zhang ${ }^{5}$, Yuting Fan ${ }^{6}$, Youcun Liu ${ }^{7}$, Zhibao Dong ${ }^{5}$, and \\ Baisheng Ye ${ }^{5, \dagger}$ \\ ${ }^{1}$ Institut de physique du globe de Paris - Sorbonne Paris Cité, Université Paris Diderot, CNRS - UMR7154, \\ 1 rue Jussieu, 75238 Paris CEDEX 05, France \\ ${ }^{2}$ Département de Géologie, CNRS - UMR8538, Ecole Normale Supérieure, 24 rue Lhomond, \\ 75005 Paris, France \\ ${ }^{3}$ Research center for Eco-environmental sciences, Chinese Academy of sciences, Beijing, P. R. China \\ ${ }^{4}$ Department of Geography, Social Science Centre Rm 2322, Western University, London, Ontario, Canada \\ ${ }^{5}$ The States Key laboratory of Cryospheric Science, Cold and Arid Region Environmental and Engineering and \\ Research Institute, Chinese Academy of Sciences, 260 Donggang west road, Lanzhou, China \\ ${ }^{6}$ Xinjiang Institute of Ecology and geography, Chinese Academy of Sciences, Urumqi, P. R. China \\ ${ }^{7}$ Key Laboratory of Water Environment and Resource, Tianjin Normal University, 393 Binshui west road, \\ Tianjin 300387, China \\ $\dagger$ deceased
}

\section{Correspondence to: François Métivier (metivier@ipgp.fr)}

Received: 12 October 2015 - Published in Earth Surf. Dynam. Discuss.: 13 November 2015

Revised: 15 February 2016 - Accepted: 9 March 2016 - Published: 22 March 2016

\begin{abstract}
The Bayanbulak Grassland, Tianshan, P. R. China, is located in an intramontane sedimentary basin where meandering and braided gravel-bed rivers coexist under the same climatic and geological settings. We report and compare measurements of the discharge, width, depth, slope and grain size of individual threads from these braided and meandering rivers. Both types of threads share statistically indistinguishable regime relations. Their depths and slopes compare well with the threshold theory, but they are wider than predicted by this theory. These findings are reminiscent of previous observations from similar gravel-bed rivers. Using the scaling laws of the threshold theory, we detrend our data with respect to discharge to produce a homogeneous statistical ensemble of width, depth and slope measurements. The statistical distributions of these dimensionless quantities are similar for braided and meandering threads. This suggests that a braided river is a collection of intertwined threads, which individually resemble those of meandering rivers. Given the environmental conditions in Bayanbulak, we furthermore hypothesize that bedload transport causes the threads to be wider than predicted by the threshold theory.
\end{abstract}

1

The morphology of alluvial rivers extends between two endmembers: in meandering rivers, the flow of water and sediments is confined in a single thread, whereas in braided rivers the flow is distributed into intertwined threads separated by bars (Fig. 1; Leopold and Wolman, 1957; Fergu- son, 1987; Ashmore, 1991; Schumm, 2005; Kleinhans and van den Berg, 2011).

Linear stability analyses, supported by laboratory experiments, explain how bedload transport generates bars, and favors the formation of meandering or braided patterns (Parker, 1976; Fredsøe, 1978; Fujita and Muramoto, 1985; Devauchelle et al., 2007; Ashmore, 1991; Zolezzi et al., 


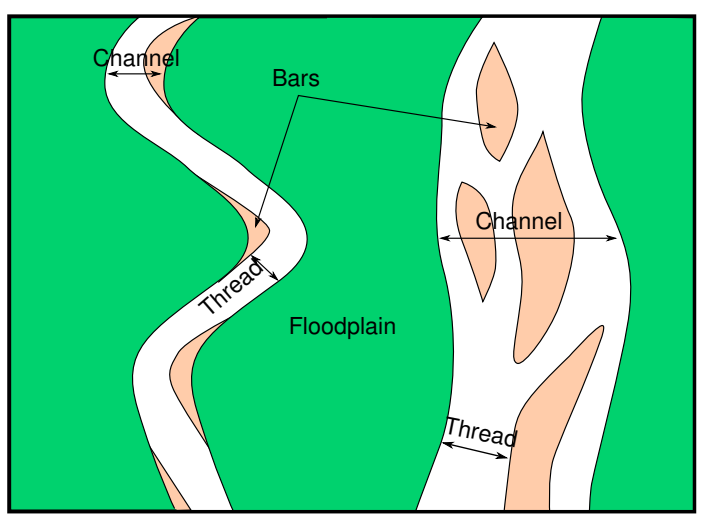

Figure 1. Definitions involved in the morphology of meandering (left) and braided (right) rivers (Métivier and Barrier, 2012).

2012). This mechanism proves more efficient in wide and shallow channels. Field measurements indicate that the bankfull aspect ratio (ratio of width to depth) of braided rivers is usually much larger than that of meandering ones, thus suggesting that the bar instability is indeed responsible for braiding (Parker, 1976; Fredsøe, 1978; Fujita and Muramoto, 1985; Devauchelle et al., 2007; Ashmore, 1991; Zolezzi et al., 2012). What exactly controls the aspect ratio of an alluvial river remains an open question, although sediment discharge and riparian vegetation seem significant in this respect: high sediment load and weak vegetation both favor wider and shallower channels, and often induce braiding (Smith and Smith, 1984; Gran and Paola, 2001; Tal and Paola, 2007, 2010; Brauderick et al., 2009; Dijk et al., 2012; Métivier and Barrier, 2012).

In a fully developed braided channel, emerged bars separate the threads from each other (Fig. 1), and the very definition of bankfull conditions becomes ambiguous. Most authors treat the channel as a whole by defining lumped quantities, such as the total channel width or the average water depth (Métivier and Barrier, 2012). Conversely, few studies focus on the morphology of braided and meandering channels at the level of individual threads (Church and Gilbert, 1975; Mosley, 1983; Ashmore, 2013; Gaurav et al., 2015). In sand-bed rivers, the geometry of braided threads appears to be indistinguishable from that of meandering ones. This observation accords with recent laboratory experiments (Seizilles et al., 2013; Reitz et al., 2014). To our knowledge, this similarity has not been fully investigated in gravel-bed rivers.

Here, we report on measurements in the Bayanbulak Grassland, Tianshan Mountains, P. R. China, where tens of meandering and braided gravel-bed rivers develop in the same environment. After comparison with other data sets from the literature, we compare the morphology of braided and meandering threads in our data set. Finally, we rescale our measurements based on the threshold theory to generate and analyze a single statistical ensemble from rivers highly

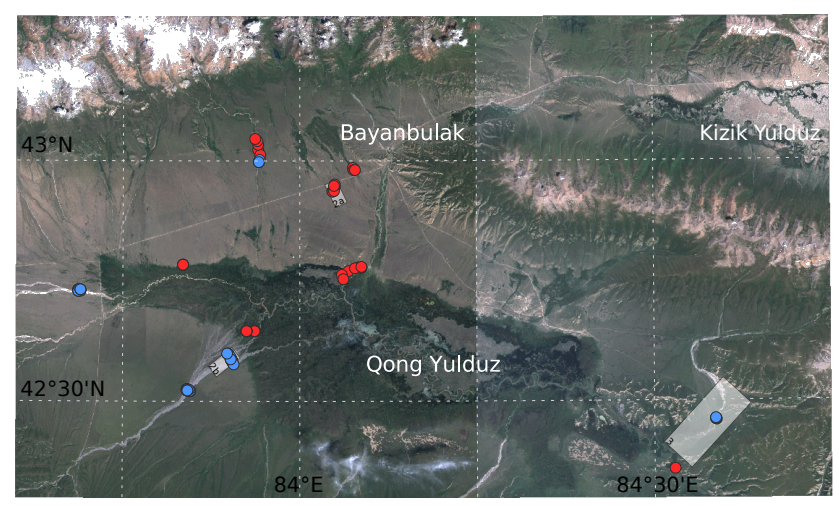

Figure 2. Landsat 5 mosaic image of the Bayanbulak Grassland. Red (meandering) and blue (braided) markers indicate measurement sites. White rectangles correspond to Figs. 4 and 5.

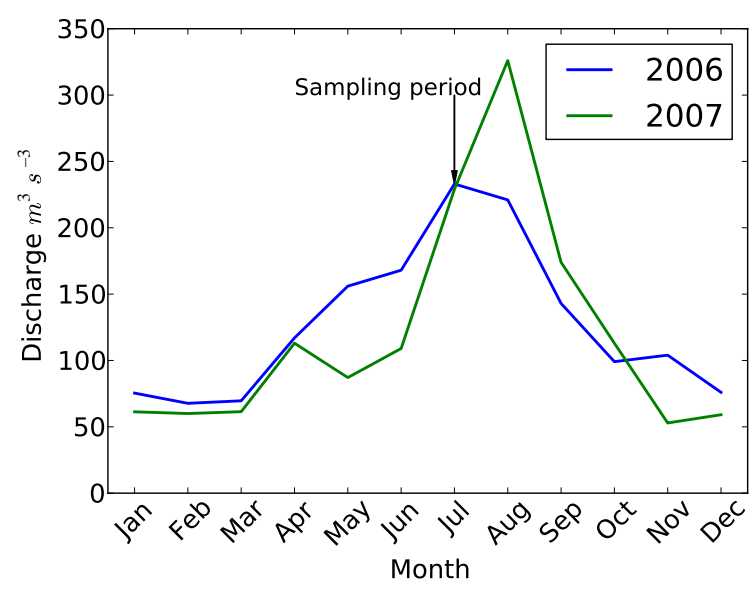

Figure 3. Water discharge of the Kaidu River at the Dashankou station, downstream of the grassland (monthly average). Source: Xinjiang Institute for Ecology and Geography (unpublished data).

dispersed in size (Glover and Florey, 1951; Henderson, 1963; Seizilles et al., 2013; Gaurav et al., 2015).

\section{Field site}

The Bayanbulak Grassland is an intramontane sedimentary basin standing at an elevation of about $2500 \mathrm{~m}$ in the Tianshan Mountains (Fig. 2). Two main wetlands, the Qong Yulduz basin (known as the Swan Lake in Chinese), and the Kizik Yulduz basin, are distributed around the main Kaidu River. They are immediately surrounded by sloping meadows (slope $S \sim 0.01$ ), themselves enclosed with the Tianshan Mountains which provide water to the Kaidu River (Zhang et al., 2002). The hydrology of the basins is controlled by snowmelt and summer orographic precipitations (Zhang et al., 2002; Yang and Cui, 2005). Snow accumulates from November to March, and starts melting in April, inducing the water discharge to rise in all rivers (Zhang et al., 2007). Orographic precipitation takes over in summer (be- 

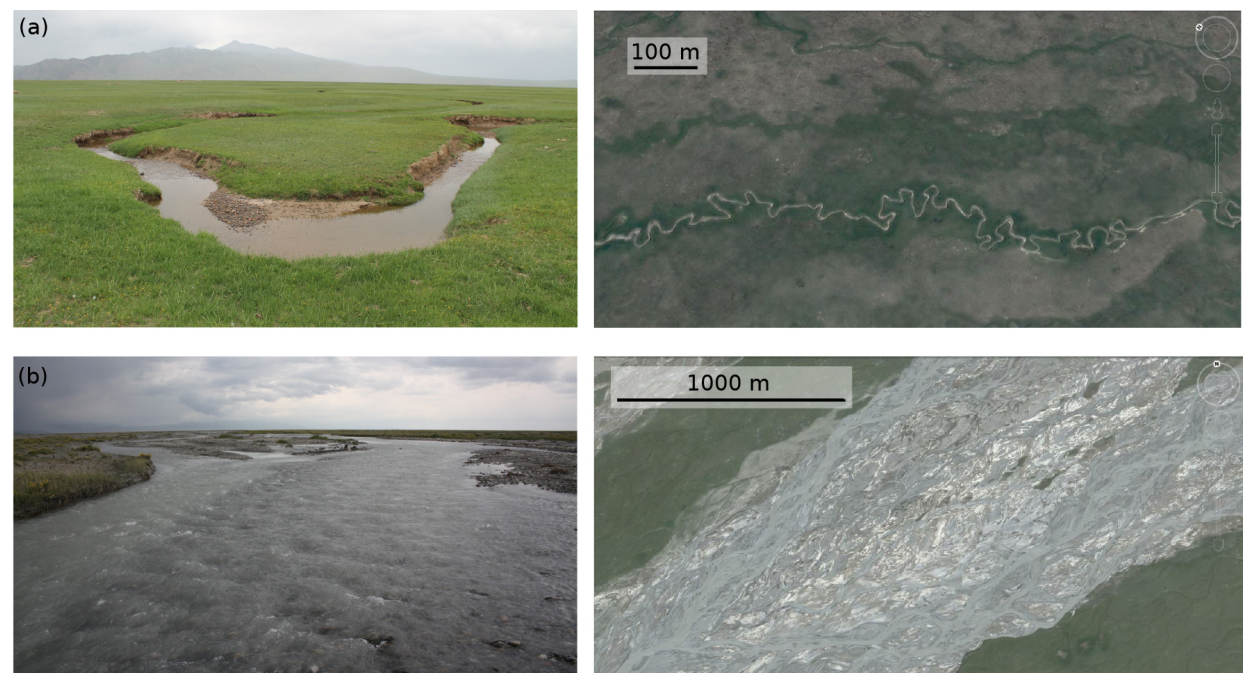

Figure 4. (a) Meandering and (b) braided rivers in the Bayanbulak Grassland. Left panels: field picture; right panels: satellite image (Google Earth). The corresponding locations also appear in Fig. 2.

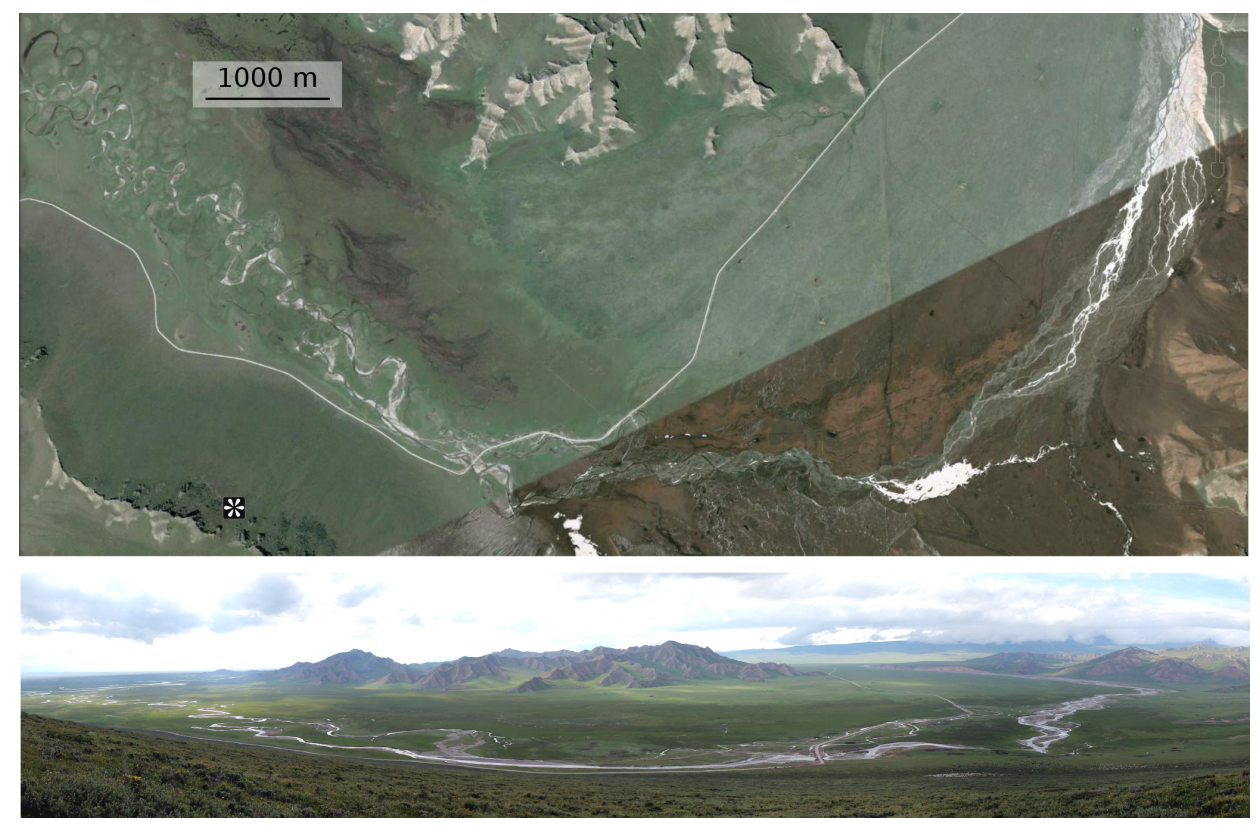

Figure 5. Satellite and panoramic view of a metamorphosis from braided to meandering (Bayanbulak Grassland, $84.578^{\circ} \mathrm{E}, 42.721^{\circ} \mathrm{N}$, Google Earth). Marker on the satellite image indicates the viewpoint of the panoramic image. Its location also appears in Fig. 2.

tween 260 and $290 \mathrm{~mm}$ ), and the discharge continues to rise until August (Fig. 3).

The morphology of the Bayanbulak rivers varies between highly meandering (sinuosity above 1.3 to 1.5 ) and braided, and the same river often switches from one to the other along its course (Figs. 4 and 5). The rivers span about 4 orders of magnitude in discharge, and about 2 in width (Fig. 6). Although a variety of grass species grow in the basin, their influence on the channel morphology is probably moderate (Zhang et al., 2002; Andrews, 1984; Métivier and Barrier,
2012). Finally, most rivers flow over gravel, whose size distribution does not vary significantly over the basin (Fig. 6). All these features combine to make the Bayanbulak Grassland an ideal field site to investigate the morphology of gravel-bed rivers.

\section{Method}

We carried out two field campaigns in July 2012 and July 2013, during the high-flow season to compare the geom- 

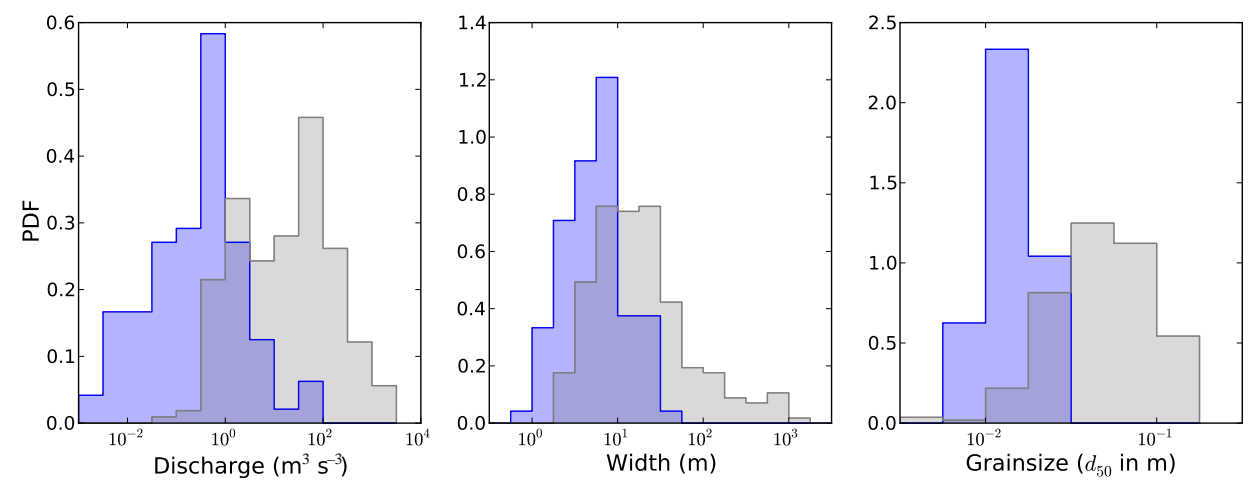

Figure 6. Normalized histograms (probability density function) of water discharge, width and grain size. Blue: this study; gray: GBR (gravel bed river) data set (Church and Rood, 1983; Parker et al., 2007; King et al., 2004; Ashmore, 2013).

etry of braided and meandering threads (Fig. 3). We treated the threads of braided rivers individually, based on the wetted area at the time of measurement (Fig. 1). We measured the cross section geometry, the discharge, the grain-size distribution and the slope of the threads from as many rivers, spanning as broad a range in discharge, as possible. We chose the sections at random, according to their accessibility, our purpose being to collect a statistically significant data set.

To measure the cross section and the water discharge of large rivers, we used a $2 \mathrm{Mhz}$ acoustic Doppler current profiler (ADCP, Teledyne-RDI StreamPro). The instrument was mounted on a raft and cross sections were performed from which we extracted both the geometry and the discharge of the threads.

In shallower rivers, we used wading rods and rulers to measure the thread geometry. The mean surface velocity was measured using floats. The average velocity was obtained from the surface velocity using a correction factor of 0.6 (Sanders, 1998; Gaurav et al., 2015). The discharge was obtained by the product of the average velocity with the wetted area.

Repeated ADCP profiles across the same section show that discharge, width and depth measurements are all reproducible within less than $15 \%$. Manual measurements yield an uncertainty of about $2 \%$ for width, $12 \%$ for depth and $25 \%$ for velocity. The resulting uncertainty on discharge is less than $40 \%$ for both methods.

We used a Topcon theodolite with a laser rangefinder to measure the long profile of the threads, and estimate their slope. The length of topographic profiles varies from $100 \mathrm{~m}$ for small braided threads to more than $3 \mathrm{~km}$ for one meandering thread. Uncertainties on the location of the theodolite and atmospheric inhomogeneities curtail the precision of long-distance profiles. For our measurements, we expect the uncertainty on angles to reach $90^{\prime \prime}$. The corresponding absolute uncertainty on the slope of a river is about $5 \times 10^{-4}$.

We measured the grain-size distribution from surface counts. Depending on the size of exposed surfaces, the number of counts ranged from 200 to 500 (Wolman, 1954; Bunte and Abt, 2001). We extracted the median grain size $d_{50}$ and the size of the 90th percentile $d_{90}$ from these distributions.

Finally, the sinuosity of the threads was measured using the topographic profiles when available. When these were not available, we used Google images and calculated the sinuosity from $1 \mathrm{~km}$ long stretches centered on the measurement site. The Bayanbulak rivers we surveyed exhibit two very distinct planforms. Single-thread rivers are, on average, highly meandering with a sinuosity of $1.5 \pm 0.2$ (Schumm, 2005). The braided rivers we surveyed have a total braiding index ranging from 3.3 to almost 11.2. As our objective is to compare these two endmembers, we ignored rivers with intermediate wandering morphology (Church, 1983). Overall, our data set is composed of 92 measurements of width, depth, average velocity, discharge, slope and grain size, among which 53 correspond to braided-river threads (Table 1), and 39 to meandering-river threads (Table 2).

\section{Regime equations}

Figure 6 compares our measurements to four other sources. Three of them, the compendiums of Parker et al. (2007), Church and Rood (1983) and King et al. (2004) include measurements from single-thread rivers. The fourth one corresponds to measurements on individual threads of the braided Sunwapta River (Ashmore, 2013). These sources are hereafter referred to as the GBR data set.

The Bayanbulak threads are widely dispersed in size $(0.6 \leq W \leq 35 \mathrm{~m})$ and discharge $\left(0.002 \leq Q \leq 51 \mathrm{~m}^{3} \mathrm{~s}^{-1}\right)$. On average, they are smaller than the GBR threads. The median grain size of the Bayanbulak threads $d_{50} \simeq 0.013 \mathrm{~m}$ is finer (the standard deviation of the $d_{50}$ is $\sigma_{d_{50}} \sim 0.008 \mathrm{~m}$ ). Our data set therefore extend the GBR ones towards smaller threads with finer sediments.

We now consider the empirical regime equations of individual threads (Fig. 7). To facilitate the comparison between the GBR data set and our own, we use dimensionless quantities, namely $W / d_{50}, H / d_{50}, S$ and $Q_{*}=Q / \sqrt{g d_{50}^{5}}$, where 
$g$ is the acceleration of gravity. Not surprisingly, the geometry of a thread is strongly correlated with its water discharge: its width and depth increase with discharge, while its slope decreases. At first sight, these trends are similar for meandering and braided threads. They also compare well to the GBR data set, although the Bayanbulak threads are slightly wider than the GBR ones on average. The measurement uncertainty, although significant, is less than the variability of our data, except for slopes smaller than about $5 \times 10^{-3}$.

Despite considerable scatter, both our measurements and the GBR data sets gather around straight lines in the log-log plots of Fig. 7, suggesting power-law regime equations:

$\frac{W}{d_{50}}=\alpha_{\mathrm{w}} Q_{*}^{\beta_{\mathrm{w}}} \quad \frac{H}{d_{50}}=\alpha_{\mathrm{h}} Q_{*}^{\beta_{\mathrm{h}}} \quad S=\alpha_{\mathrm{s}} Q_{*}^{\beta_{\mathrm{s}}}$,

where $\alpha_{\mathrm{w}}, \alpha_{\mathrm{h}}, \alpha_{\mathrm{s}}, \beta_{\mathrm{w}}, \beta_{\mathrm{h}}$ and $\beta_{\mathrm{s}}$ are dimensionless parameters. To evaluate them, we use reduced major axis regression (RMA) instead of least square regression because the variability of our data is comparable along both axes (Sokal and Rohlf, 1995; Scherrer, 1984). The resulting fitted coefficients are reported in Table 3. The scatter in the slope measurement is too large to provide significant estimates of the slope coefficients $\alpha_{\mathrm{s}}$ and $\beta_{\mathrm{s}}$. At the $95 \%$ confidence level, the regime relationships of meandering and braided threads cannot be distinguished. Similarly, the depth of the Bayanbulak threads cannot be distinguished from those of the GBR threads. Conversely, the Bayanbulak threads are significantly wider than the GBR threads with respect to their median grain size.

So far we have made the width, depth and discharge dimensionless using $d_{50}$ as the characteristic grain size of the sediment. This choice, however, is arbitrary (Parker et al., 2007; Parker, 2008). Large grains are arguably more likely to control the geometry of the threads than smaller ones, and a larger quantile might be a better approximation of the characteristic grain size. For comparison, we rescaled our measurements using $d_{90}$ instead of $d_{50}$, and repeated the above analysis. Our conclusions are not altered significantly by this choice of characteristic grain size (Table 3).

\section{Detrending}

So far, we have found that the empirical regime equations of meandering and braided threads are statistically similar. To proceed further with this comparison, we would like to convert our measurements into a single statistical ensemble. We thus need to detrend our data set with respect to water discharge, based on analytical regime equations. Following Gaurav et al. (2015), we propose to use the threshold theory to do so.

The threshold theory assumes that a river transports its sediment load slowly enough for its bed to be near the threshold of motion (Glover and Florey, 1951; Henderson, 1963; Yalin and Ferreira da Silva, 2001; Seizilles,
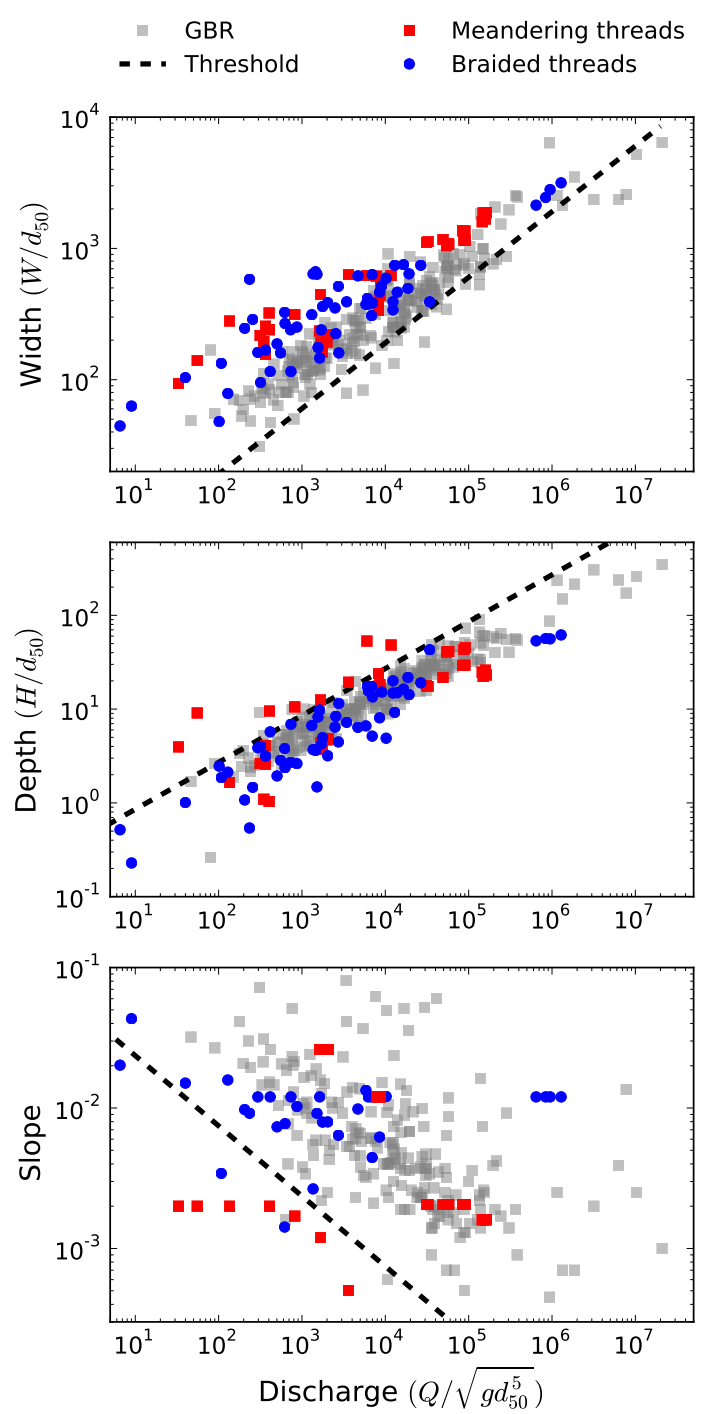

Figure 7. Dimensionless width, depth and slope of individual gravel-bed threads as a function of dimensionless water discharge. Dashed lines represent the threshold theory.

2013). Momentum and mass balances then yield power-law regime equations, the original formulation of which reads (Glover and Florey, 1951)

$$
\begin{aligned}
& \frac{W}{d_{\mathrm{s}}}=\left[\frac{\pi}{\sqrt{\mu}}\left(\frac{\theta_{\mathrm{t}}\left(\rho_{\mathrm{s}}-\rho\right)}{\rho}\right)^{-1 / 4} \sqrt{\frac{3 C_{\mathrm{f}}}{2^{3 / 2} \mathcal{K}[1 / 2]}}\right] Q_{*}^{1 / 2}, \\
& \frac{H}{d_{\mathrm{s}}}=\left[\frac{\sqrt{\mu}}{\pi}\left(\frac{\theta_{\mathrm{t}}\left(\rho_{\mathrm{s}}-\rho\right)}{\rho}\right)^{-1 / 4} \sqrt{\frac{3 \sqrt{2} C_{\mathrm{f}}}{\mathcal{K}[1 / 2]}}\right] Q_{*}^{1 / 2}, \\
& S=\left[\left(\mu^{1 / 2} \frac{\theta_{\mathrm{t}}\left(\rho_{\mathrm{s}}-\rho\right)}{\rho}\right)^{5 / 4} \sqrt{\frac{\mathcal{K}[1 / 2] 2^{3 / 2}}{3 C_{\mathrm{f}}}}\right] Q_{*}^{-1 / 2},
\end{aligned}
$$

where 
Table 1. Data gathered for braided-river threads. Latitude (lat) and longitude (long) are in degrees centesimal; measurement stands for measurement type (Fl: float, ADCP: acoustic Doppler current profiler); $Q$ : discharge, Sec: wetted area, $V:$ average velocity, $W$ : width, $H$ : depth, $d_{50}$ : median grain size, $d_{90}$ : size of the 90 th percentile, $S$ : slope. All physical quantities are given in the International System of Units.

\begin{tabular}{|c|c|c|c|c|c|c|c|c|c|c|c|c|}
\hline Code & Lat & Long & Channel & Measurement & $Q$ & $\mathrm{Sec}$ & $V$ & $W$ & $H$ & $d_{50}$ & $d_{90}$ & $S$ \\
\hline 174 & 42.7611 & 83.841 & B & $\mathrm{Fl}$ & 2.9 & 2.6 & 1.1 & 18.0 & 0.14 & 0.028 & 0.064 & 0.0044 \\
\hline 180 & 42.7617 & 83.8428 & $\mathrm{~B}$ & $\mathrm{Fl}$ & 4.2 & 2.3 & 1.8 & 17.0 & 0.14 & 0.028 & 0.064 & 0.012 \\
\hline 181 & 42.7602 & 83.8454 & $\mathrm{~B}$ & $\mathrm{Fl}$ & 1.1 & 1.8 & 0.62 & 14.0 & 0.13 & 0.028 & 0.064 & 0.0064 \\
\hline 182 & 42.7604 & 83.8448 & $\mathrm{~B}$ & $\mathrm{Fl}$ & 0.36 & 0.52 & 0.69 & 7.0 & 0.073 & 0.028 & 0.064 & 0.01 \\
\hline 183 & 42.7617 & 83.8439 & B & $\mathrm{Fl}$ & 0.73 & 1.4 & 0.52 & 10.0 & 0.14 & 0.028 & 0.064 & 0.0079 \\
\hline 184 & 42.7609 & 83.8417 & $\mathrm{~B}$ & $\mathrm{Fl}$ & 0.62 & 0.74 & 0.84 & 18.0 & 0.041 & 0.028 & 0.064 & 0.0092 \\
\hline 185 & 42.7608 & 83.8415 & B & $\mathrm{Fl}$ & 2.4 & 1.9 & 1.2 & 11.0 & 0.19 & 0.028 & 0.064 & 0.013 \\
\hline 168 & 42.7307 & 84.5878 & $\mathrm{~B}$ & $\mathrm{Fl}$ & 0.0034 & 0.011 & 0.32 & 1.7 & 0.0062 & 0.027 & 0.064 & 0.043 \\
\hline 142 & 42.9985 & 83.943 & $\mathrm{~B}$ & $\mathrm{Fl}$ & 0.025 & 0.11 & 0.23 & 1.5 & 0.074 & 0.013 & 0.064 & 0.012 \\
\hline 141 & 43.0004 & 83.9435 & B & $\mathrm{Fl}$ & 0.38 & 1.0 & 0.38 & 4.9 & 0.2 & 0.013 & 0.064 & 0.012 \\
\hline 139 & 42.9985 & 83.943 & B & $\mathrm{Fl}$ & 0.41 & 0.9 & 0.46 & 4.0 & 0.23 & 0.013 & 0.064 & 0.012 \\
\hline 137 & 43.0004 & 83.9435 & B & $\mathrm{Fl}$ & 0.099 & 0.24 & 0.41 & 1.9 & 0.13 & 0.013 & 0.064 & 0.012 \\
\hline 179 & 42.7618 & 83.8416 & B & $\mathrm{Fl}$ & 0.84 & 0.96 & 0.87 & 11.0 & 0.089 & 0.028 & 0.064 & 0.008 \\
\hline 178 & 42.7609 & 83.8413 & B & $\mathrm{Fl}$ & 0.097 & 0.25 & 0.39 & 16.0 & 0.015 & 0.028 & 0.064 & 0.0092 \\
\hline 173 & 42.7622 & 83.8404 & B & $\mathrm{Fl}$ & 0.56 & 1.9 & 0.3 & 18.0 & 0.1 & 0.028 & 0.064 & 0.0027 \\
\hline 172 & 42.7605 & 83.8447 & B & $\mathrm{Fl}$ & 0.21 & 0.28 & 0.72 & 5.3 & 0.054 & 0.028 & 0.064 & 0.0073 \\
\hline 171 & 42.7608 & 83.8446 & B & $\mathrm{Fl}$ & 0.26 & 0.5 & 0.52 & 7.5 & 0.066 & 0.028 & 0.064 & 0.0077 \\
\hline 170 & 42.7619 & 83.8416 & B & $\mathrm{Fl}$ & 1.9 & 3.1 & 0.62 & 17.0 & 0.18 & 0.028 & 0.064 & 0.0098 \\
\hline 169 & 42.7316 & 84.5874 & B & $\mathrm{Fl}$ & 0.23 & 0.9 & 0.26 & 8.8 & 0.1 & 0.027 & 0.064 & 0.0014 \\
\hline 175 & 42.7604 & 83.8452 & B & $\mathrm{Fl}$ & 0.084 & 0.21 & 0.41 & 6.9 & 0.03 & 0.028 & 0.064 & 0.0097 \\
\hline 167 & 42.7314 & 84.5877 & B & $\mathrm{Fl}$ & 0.0025 & 0.017 & 0.15 & 1.2 & 0.014 & 0.027 & 0.064 & 0.02 \\
\hline 166 & 42.731 & 84.5877 & B & $\mathrm{Fl}$ & 0.015 & 0.076 & 0.2 & 2.8 & 0.027 & 0.027 & 0.064 & 0.015 \\
\hline 165 & 42.7305 & 84.5887 & B & $\mathrm{Fl}$ & 0.04 & 0.18 & 0.22 & 3.6 & 0.05 & 0.027 & 0.064 & 0.0034 \\
\hline 176 & 42.7609 & 83.8419 & B & $\mathrm{Fl}$ & 3.5 & 2.9 & 1.2 & 13.0 & 0.23 & 0.028 & 0.064 & 0.0062 \\
\hline 177 & 42.7623 & 83.8404 & B & $\mathrm{Fl}$ & 0.053 & 0.13 & 0.4 & 2.2 & 0.06 & 0.028 & 0.064 & 0.016 \\
\hline 135 & 43.0004 & 83.9435 & B & $\mathrm{Fl}$ & 0.018 & 0.11 & 0.17 & 2.1 & 0.05 & 0.013 & 0.064 & 0.012 \\
\hline 134 & 42.9985 & 83.943 & B & $\mathrm{Fl}$ & 0.044 & 0.13 & 0.33 & 1.5 & 0.09 & 0.013 & 0.064 & 0.012 \\
\hline 125 & 42.7995 & 83.8983 & B & Fl & 0.19 & 0.44 & 0.43 & 4.9 & 0.09 & 0.013 & 0.053 & 0.008 \\
\hline 111 & 42.7898 & 83.9064 & B & $\mathrm{Fl}$ & 0.03 & 0.071 & 0.43 & 2.0 & 0.036 & 0.013 & 0.053 & 0.008 \\
\hline 112 & 42.7944 & 83.9025 & B & $\mathrm{Fl}$ & 1.9 & 2.6 & 0.71 & 4.9 & 0.54 & 0.013 & 0.053 & 0.008 \\
\hline 113 & 42.7946 & 83.9025 & B & $\mathrm{Fl}$ & 0.14 & 0.29 & 0.47 & 2.8 & 0.1 & 0.013 & 0.053 & 0.008 \\
\hline 114 & 42.7985 & 83.8993 & B & $\mathrm{Fl}$ & 1.0 & 1.7 & 0.61 & 6.2 & 0.27 & 0.013 & 0.053 & 0.008 \\
\hline 115 & 42.799 & 83.8992 & B & $\mathrm{Fl}$ & 0.15 & 0.29 & 0.53 & 2.0 & 0.14 & 0.013 & 0.053 & 0.008 \\
\hline 116 & 42.7924 & 83.904 & B & $\mathrm{Fl}$ & 0.0056 & 0.018 & 0.3 & 0.6 & 0.031 & 0.013 & 0.053 & 0.008 \\
\hline 117 & 42.7911 & 83.9053 & B & $\mathrm{Fl}$ & 0.02 & 0.083 & 0.24 & 2.1 & 0.04 & 0.013 & 0.053 & 0.008 \\
\hline 118 & 42.7991 & 83.8989 & B & $\mathrm{Fl}$ & 0.39 & 0.81 & 0.48 & 4.8 & 0.17 & 0.013 & 0.053 & 0.008 \\
\hline 110 & 42.7983 & 83.8995 & B & $\mathrm{Fl}$ & 0.68 & 0.91 & 0.74 & 4.9 & 0.19 & 0.013 & 0.053 & 0.008 \\
\hline 109 & 42.7914 & 83.905 & B & $\mathrm{Fl}$ & 0.14 & 0.35 & 0.39 & 4.4 & 0.08 & 0.013 & 0.053 & 0.008 \\
\hline 101 & 42.7915 & 83.905 & B & $\mathrm{Fl}$ & 0.71 & 1.1 & 0.66 & 9.3 & 0.12 & 0.013 & 0.053 & 0.008 \\
\hline 102 & 42.7921 & 83.9042 & B & $\mathrm{Fl}$ & 0.91 & 1.9 & 0.47 & 9.4 & 0.2 & 0.013 & 0.053 & 0.008 \\
\hline 103 & 42.7944 & 83.9025 & B & $\mathrm{Fl}$ & 0.04 & 0.1 & 0.39 & 3.0 & 0.034 & 0.013 & 0.053 & 0.008 \\
\hline 104 & 42.7946 & 83.9025 & B & $\mathrm{Fl}$ & 0.093 & 0.16 & 0.59 & 3.0 & 0.053 & 0.013 & 0.053 & 0.008 \\
\hline 105 & 42.7916 & 83.9047 & B & $\mathrm{Fl}$ & 0.014 & 0.066 & 0.21 & 3.6 & 0.018 & 0.013 & 0.053 & 0.008 \\
\hline 106 & 42.7985 & 83.8994 & B & $\mathrm{Fl}$ & 0.08 & 0.38 & 0.21 & 8.3 & 0.045 & 0.013 & 0.053 & 0.008 \\
\hline 107 & 42.7983 & 83.8994 & B & $\mathrm{Fl}$ & 0.76 & 1.1 & 0.7 & 5.8 & 0.19 & 0.013 & 0.053 & 0.008 \\
\hline 108 & 42.7898 & 83.9063 & B & $\mathrm{Fl}$ & 1.1 & 1.4 & 0.74 & 8.0 & 0.18 & 0.013 & 0.053 & 0.008 \\
\hline 119 & 42.7925 & 83.9037 & B & $\mathrm{Fl}$ & 0.017 & 0.06 & 0.29 & 1.2 & 0.05 & 0.013 & 0.053 & 0.008 \\
\hline 100 & 42.7925 & 83.9037 & B & $\mathrm{Fl}$ & 0.085 & 0.23 & 0.38 & 2.2 & 0.1 & 0.013 & 0.053 & 0.008 \\
\hline 124 & 42.7934 & 83.903 & B & $\mathrm{Fl}$ & 0.5 & 1.2 & 0.4 & 6.5 & 0.19 & 0.013 & 0.053 & 0.008 \\
\hline 123 & 42.7884 & 83.907 & B & $\mathrm{Fl}$ & 0.072 & 0.33 & 0.22 & 3.9 & 0.083 & 0.013 & 0.053 & 0.008 \\
\hline 122 & 42.7926 & 83.9037 & B & $\mathrm{Fl}$ & 0.68 & 1.1 & 0.64 & 4.3 & 0.25 & 0.013 & 0.053 & 0.008 \\
\hline 121 & 42.7937 & 83.9028 & B & $\mathrm{Fl}$ & 1.5 & 2.2 & 0.66 & 9.3 & 0.24 & 0.013 & 0.053 & 0.008 \\
\hline 120 & 42.7953 & 83.9025 & B & $\mathrm{Fl}$ & 0.33 & 1.1 & 0.29 & 5.2 & 0.22 & 0.013 & 0.053 & 0.008 \\
\hline 646 & 42.6926 & 83.6944 & B & $\mathrm{ADCP}$ & 51.0 & 24.0 & 2.2 & 35.0 & 0.68 & 0.011 & 0.15 & 0.012 \\
\hline 649 & 42.6926 & 83.6944 & B & ADCP & 33.0 & 17.0 & 2.0 & 27.0 & 0.62 & 0.011 & 0.15 & 0.012 \\
\hline 652 & 42.6926 & 83.6944 & B & $\mathrm{ADCP}$ & 26.0 & 14.0 & 1.9 & 23.0 & 0.59 & 0.011 & 0.15 & 0.012 \\
\hline 655 & 42.6926 & 83.6944 & B & ADCP & 38.0 & 19.0 & 2.0 & 31.0 & 0.62 & 0.011 & 0.15 & 0.012 \\
\hline
\end{tabular}


Table 2. Data gathered for meandering-river threads. Latitude (lat) and longitude (long) are in degrees centesimal; measurement stands for measurement type (Fl: float, ADCP: acoustic doppler current profiler); $Q$ : discharge, Sec: wetted area, $V$ : average velocity, $W$ : width, $H$ : depth, $d_{50}$ : median grain size, $d_{90}$ : size of the 90 th percentile, $S$ : slope. All physical quantities are given in the International System of Units.

\begin{tabular}{|c|c|c|c|c|c|c|c|c|c|c|c|c|}
\hline Code & Lat & Long & Channel & Measurement & $Q$ & $\mathrm{Sec}$ & $V$ & $W$ & $H$ & $d_{50}$ & $d_{90}$ & $S$ \\
\hline 614 & 42.8229 & 83.9253 & M & $\mathrm{ADCP}$ & 0.69 & 2.1 & 0.33 & 7.3 & 0.28 & 0.007 & 0.038 & 0.0021 \\
\hline 626 & 42.8915 & 83.835 & M & $\mathrm{ADCP}$ & 8.2 & 6.5 & 1.3 & 23.0 & 0.28 & 0.013 & 0.03 & 0.0016 \\
\hline 609 & 42.8227 & 83.9366 & M & $\mathrm{ADCP}$ & 1.1 & 1.9 & 0.56 & 9.5 & 0.2 & 0.007 & 0.038 & 0.0021 \\
\hline 625 & 42.8915 & 83.835 & M & $\mathrm{ADCP}$ & 8.9 & 6.7 & 1.3 & 23.0 & 0.29 & 0.013 & 0.03 & 0.0016 \\
\hline 610 & 42.8227 & 83.9366 & M & $\mathrm{ADCP}$ & 1.2 & 2.0 & 0.59 & 9.5 & 0.21 & 0.007 & 0.038 & 0.0021 \\
\hline 624 & 42.8915 & 83.835 & M & $\mathrm{ADCP}$ & 7.9 & 6.2 & 1.3 & 20.0 & 0.31 & 0.013 & 0.03 & 0.0016 \\
\hline 611 & 42.8227 & 83.9366 & M & $\mathrm{ADCP}$ & 1.1 & 2.4 & 0.46 & 8.1 & 0.3 & 0.007 & 0.038 & 0.0021 \\
\hline 612 & 42.8227 & 83.9366 & M & $\mathrm{ADCP}$ & 1.2 & 2.5 & 0.46 & 8.0 & 0.32 & 0.007 & 0.038 & 0.0021 \\
\hline 623 & 42.8915 & 83.835 & M & $\mathrm{ADCP}$ & 8.7 & 6.8 & 1.3 & 21.0 & 0.33 & 0.013 & 0.03 & 0.0016 \\
\hline 613 & 42.8229 & 83.9253 & M & $\mathrm{ADCP}$ & 0.74 & 2.2 & 0.34 & 7.5 & 0.29 & 0.007 & 0.038 & 0.0021 \\
\hline 617 & 42.8229 & 83.9253 & M & $\mathrm{ADCP}$ & 0.42 & 0.97 & 0.43 & 7.9 & 0.12 & 0.007 & 0.038 & 0.0021 \\
\hline 616 & 42.8229 & 83.9253 & M & $\mathrm{ADCP}$ & 0.4 & 0.96 & 0.42 & 7.8 & 0.12 & 0.007 & 0.038 & 0.0021 \\
\hline 615 & 42.8229 & 83.9253 & M & $\mathrm{ADCP}$ & 0.73 & 2.2 & 0.33 & 7.6 & 0.29 & 0.007 & 0.038 & 0.0021 \\
\hline 608 & 42.8227 & 83.9366 & M & $\mathrm{ADCP}$ & 0.63 & 1.2 & 0.51 & 8.2 & 0.15 & 0.007 & 0.038 & 0.0021 \\
\hline 144 & 43.0224 & 83.9376 & M & $\mathrm{Fl}$ & 0.53 & 1.3 & 0.4 & 6.6 & 0.2 & 0.013 & 0.064 & 0.012 \\
\hline 151 & 42.9721 & 84.0495 & M & $\mathrm{Fl}$ & 0.0076 & 0.046 & 0.16 & 1.9 & 0.024 & 0.009 & 0.034 & 0.01 \\
\hline 150 & 42.9901 & 84.0785 & M & $\mathrm{Fl}$ & 0.3 & 0.32 & 0.94 & 3.6 & 0.088 & 0.02 & 0.014 & 0.026 \\
\hline 149 & 42.9902 & 84.0764 & M & $\mathrm{Fl}$ & 0.3 & 0.34 & 0.88 & 3.9 & 0.088 & 0.02 & 0.014 & 0.026 \\
\hline 148 & 42.9925 & 84.0758 & M & $\mathrm{Fl}$ & 0.37 & 0.42 & 0.87 & 4.4 & 0.096 & 0.02 & 0.014 & 0.026 \\
\hline 147 & 42.9909 & 84.0781 & M & $\mathrm{Fl}$ & 0.29 & 0.35 & 0.82 & 4.7 & 0.074 & 0.02 & 0.014 & 0.026 \\
\hline 146 & 42.9679 & 84.0473 & M & $\mathrm{Fl}$ & 0.18 & 0.16 & 1.1 & 2.6 & 0.061 & 0.016 & 0.04 & 0.012 \\
\hline 145 & 42.9682 & 84.0468 & M & $\mathrm{Fl}$ & 0.2 & 0.23 & 0.87 & 3.1 & 0.075 & 0.016 & 0.04 & 0.012 \\
\hline 143 & 43.0206 & 83.9402 & M & $\mathrm{Fl}$ & 0.5 & 1.1 & 0.45 & 4.9 & 0.23 & 0.013 & 0.064 & 0.012 \\
\hline 140 & 43.0167 & 83.9418 & M & $\mathrm{Fl}$ & 0.5 & 1.4 & 0.37 & 4.4 & 0.31 & 0.013 & 0.064 & 0.012 \\
\hline 138 & 43.0059 & 83.945 & M & $\mathrm{Fl}$ & 0.47 & 1.6 & 0.29 & 8.0 & 0.21 & 0.013 & 0.064 & 0.012 \\
\hline 136 & 43.011 & 83.9416 & M & $\mathrm{Fl}$ & 0.52 & 1.4 & 0.39 & 5.7 & 0.24 & 0.013 & 0.064 & 0.012 \\
\hline 152 & 42.9713 & 84.049 & M & $\mathrm{Fl}$ & 0.0088 & 0.053 & 0.17 & 2.3 & 0.023 & 0.009 & 0.034 & 0.01 \\
\hline 153 & 42.9751 & 84.0496 & M & $\mathrm{Fl}$ & 0.0088 & 0.052 & 0.17 & 1.4 & 0.037 & 0.009 & 0.034 & 0.01 \\
\hline 164 & 42.8769 & 84.0626 & $\mathrm{M}$ & $\mathrm{Fl}$ & 0.52 & 7.4 & 0.07 & 9.3 & 0.8 & 0.015 & 0.034 & 0.00015 \\
\hline 163 & 42.8895 & 84.0873 & $\mathrm{M}$ & $\mathrm{Fl}$ & 0.012 & 0.1 & 0.11 & 4.2 & 0.025 & 0.015 & 0.034 & 0.002 \\
\hline 162 & 42.8881 & 84.0782 & $\mathrm{M}$ & $\mathrm{Fl}$ & 0.14 & 1.3 & 0.11 & 6.7 & 0.19 & 0.015 & 0.034 & 0.0012 \\
\hline 161 & 42.8812 & 84.0603 & M & $\mathrm{Fl}$ & 1.0 & 6.7 & 0.15 & 9.3 & 0.72 & 0.015 & 0.034 & 0.00015 \\
\hline 160 & 42.8887 & 84.0836 & $\mathrm{M}$ & $\mathrm{Fl}$ & 0.071 & 0.74 & 0.095 & 4.7 & 0.16 & 0.015 & 0.034 & 0.0017 \\
\hline 159 & 42.889 & 84.0881 & M & $\mathrm{Fl}$ & 0.0029 & 0.083 & 0.035 & 1.4 & 0.059 & 0.015 & 0.034 & 0.002 \\
\hline 158 & 42.8852 & 84.0688 & M & $\mathrm{Fl}$ & 0.31 & 2.8 & 0.11 & 9.5 & 0.29 & 0.015 & 0.034 & 0.0005 \\
\hline 157 & 42.889 & 84.0861 & M & $\mathrm{Fl}$ & 0.035 & 0.52 & 0.068 & 3.6 & 0.14 & 0.015 & 0.034 & 0.002 \\
\hline 156 & 42.8891 & 84.088 & $\mathrm{M}$ & $\mathrm{Fl}$ & 0.0048 & 0.29 & 0.017 & 2.1 & 0.14 & 0.015 & 0.034 & 0.002 \\
\hline 155 & 42.9733 & 84.0494 & $\mathrm{M}$ & $\mathrm{Fl}$ & 0.0084 & 0.017 & 0.49 & 1.8 & 0.0099 & 0.009 & 0.034 & 0.01 \\
\hline 154 & 42.9686 & 84.0489 & M & $\mathrm{Fl}$ & 0.0097 & 0.027 & 0.36 & 2.9 & 0.0093 & 0.009 & 0.034 & 0.01 \\
\hline
\end{tabular}

$Q_{*}=\frac{Q}{\sqrt{g d_{\mathrm{s}}^{5}}}$

is the dimensionless discharge. $\rho=1000 \mathrm{~kg} \mathrm{~m}^{-3}$ and $\rho_{\mathrm{S}}=2650 \mathrm{~kg} \mathrm{~m}^{-3}$ are the densities of water and sediment, $C_{\mathrm{f}} \approx 0.1$ is the turbulent friction coefficient, $Q$ the water discharge, $\theta_{\mathrm{t}} \sim 0.04$ the threshold Shields parameter, $\mu \sim 0.7$ the friction angle for gravel, and $\mathcal{K}[1 / 2] \approx 1.85$ a transcendental integral (Glover and Florey, 1951; Henderson, 1963; Seizilles et al., 2013).
This formulation is similar to the one proposed by Parker et al. (2007), but for two points. First, Eqs. (2) to (4) represent a threshold channel, whereas Parker et al. (2007) extend the theory to active channels. Second, the formulation of Glover and Florey (1951) uses a constant friction coefficient in the momentum balance, whereas Parker et al. (2007) use a more elaborate friction law. Here we use the simplest formulation, as the variability of our data overshadows these differences (Métivier and Barrier, 2012). 
Table 3. Linear regressions on the $\log _{10}$ of width and depth as functions of discharge and for two characteristic grain sizes. The confidence level is $95 \%$. RMA: reduced major axis regression $\sigma_{\beta}$ stands for confidence interval on the slope of the regression $\beta$.

\begin{tabular}{|c|c|c|c|c|c|}
\hline \multicolumn{6}{|c|}{ Width: $\log _{10}\left(W / d_{\mathrm{S}}\right)=\beta_{\mathrm{W}} \log _{10} Q_{*}+\alpha_{\mathrm{W}}$} \\
\hline Thread & $d_{\mathrm{S}}$ & $\beta_{\mathrm{w}}$ & $\alpha_{\mathrm{w}}$ & $\sigma_{\beta_{\mathrm{w}}}$ & $R^{2}$ \\
\hline \multirow[t]{2}{*}{ Total pop. } & $d_{50}$ & 0.33 & 1.47 & 0.03 & 0.83 \\
\hline & $d_{90}$ & 0.34 & 1.35 & 0.04 & 0.78 \\
\hline \multirow[t]{2}{*}{ Meandering } & $d_{50}$ & 0.35 & 1.38 & 0.04 & 0.91 \\
\hline & $d_{90}$ & 0.33 & 1.37 & 0.04 & 0.91 \\
\hline \multirow[t]{2}{*}{ Braided } & $d_{50}$ & 0.32 & 1.51 & 0.05 & 0.78 \\
\hline & $d_{90}$ & 0.35 & 1.34 & 0.07 & 0.66 \\
\hline \multirow[t]{2}{*}{ GBR } & $d_{50}$ & 0.41 & 0.86 & 0.02 & 0.87 \\
\hline & $d_{90}$ & 0.49 & 0.78 & 0.04 & 0.94 \\
\hline \multicolumn{6}{|c|}{ Depth: $\log _{10}\left(H / d_{\mathrm{s}}\right)=\beta_{\mathrm{h}} \log _{10} Q_{*}+\alpha_{\mathrm{h}}$} \\
\hline Thread type & $d_{\mathrm{s}}$ & $\beta_{\mathrm{h}}$ & $\alpha_{\mathrm{h}}$ & $\sigma_{\beta_{\mathrm{h}}}$ & $R^{2}$ \\
\hline \multirow[t]{2}{*}{ Total pop. } & $d_{50}$ & 0.44 & -0.62 & 0.04 & 0.84 \\
\hline & $d_{90}$ & 0.4 & -0.5 & 0.05 & 0.77 \\
\hline \multirow[t]{2}{*}{ Meandering } & $d_{50}$ & 0.44 & -0.66 & 0.08 & 0.84 \\
\hline & $d_{90}$ & 0.39 & -0.46 & 0.08 & 0.8 \\
\hline \multirow[t]{2}{*}{ Braided } & $d_{50}$ & 0.44 & -0.61 & 0.05 & 0.83 \\
\hline & $d_{90}$ & 0.41 & -0.51 & 0.07 & 0.7 \\
\hline \multirow[t]{2}{*}{ GBR } & $d_{50}$ & 0.45 & -0.63 & 0.02 & 0.92 \\
\hline & $d_{90}$ & 0.34 & -0.29 & 0.04 & 0.9 \\
\hline
\end{tabular}

The dashed lines on Fig. 7 represent Eqs. (2) to (4). On average, the Bayanbulak threads are wider, shallower and steeper than the corresponding threshold thread. However, the theory predicts reasonably their dependence with respect to discharge, thus supporting its use to detrend our data. Accordingly, we define a set of rescaled quantities as follows:

$$
\begin{aligned}
& W_{*}=\frac{W}{d_{\mathrm{s}} C_{\mathrm{W}} \sqrt{Q_{*}}}=\frac{W\left(g d_{\mathrm{s}}\right)^{1 / 4}}{C_{\mathrm{W}} \sqrt{Q}}, \\
& H_{*}=\frac{H}{d_{\mathrm{s}} C_{\mathrm{H}} \sqrt{Q_{*}}}=\frac{H\left(g d_{\mathrm{s}}\right)^{1 / 4}}{C_{\mathrm{H}} \sqrt{Q}}, \\
& S_{*}=S \frac{\sqrt{Q_{*}}}{C_{\mathrm{S}}}=\frac{S \sqrt{Q}}{g^{1 / 4} d_{\mathrm{s}}^{5 / 4} C_{\mathrm{S}}} .
\end{aligned}
$$

Here the coefficients $C_{\mathrm{W}}, C_{\mathrm{H}}, C_{\mathrm{S}}$ correspond to the prefactors in square brackets of Eqs. (2) to (4). We used the typical values reported above for the coefficients that do not vary in our data set.

Figure 8 shows the relationship between the rescaled thread geometry and its dimensionless discharge, using $d_{50}$ to approximate the characteristic grain size $d_{\mathrm{s}}$. The new quantities $W_{*}, H_{*}$ and $S_{*}$ appear far less dependent on the water discharge than their original counterpart, although a residual trend remains for all of them. Using ordinary least squares, we fit power laws to our rescaled data to evaluate this residual trend. We find $W_{*} \propto Q_{*}^{-0.19 \pm 0.03}$ and $H_{*} \propto Q_{*}^{-0.10 \pm 0.05}$ for the Bayanbulak threads, and
Table 4. Mean and standard deviations of the logarithms of detrended widths, depths and slopes. The aspect ratios is naturally detrended and does not depend on grain size.

\begin{tabular}{lcrl}
\hline River type & Grain size & Mean & SD \\
\hline \multicolumn{4}{c}{ Width $\log _{10}\left(W_{*}\right)$} \\
\hline Meandering & $d_{50}$ & 0.6 & 0.3 \\
Meandering & $d_{90}$ & 0.8 & 0.2 \\
Braided & $d_{50}$ & 0.7 & 0.3 \\
Braided & $d_{90}$ & 0.8 & 0.2 \\
GBR & $d_{50}$ & 0.3 & 0.2 \\
GBR & $d_{90}$ & 0.5 & 0.1 \\
\hline \multicolumn{5}{c}{ Depth $\log _{10}\left(H_{*}\right)$} \\
\hline Meandering & $d_{50}$ & -0.1 & 0.3 \\
Meandering & $d_{90}$ & -0.0 & 0.3 \\
Braided & $d_{50}$ & -0.2 & 0.2 \\
Braided & $d_{90}$ & -0.1 & 0.2 \\
GBR & $d_{50}$ & -0.2 & 0.1 \\
GBR & $d_{90}$ & -0.0 & 0.2 \\
\hline \multicolumn{5}{c}{ Slope $\log _{10}\left(S_{*}\right)$} \\
\hline Meandering & $d_{50}$ & 0.4 & 0.7 \\
Meandering & $d_{90}$ & -0.22 & 0.74 \\
Braided & $d_{50}$ & 0.5 & 0.7 \\
Braided & $d_{90}$ & -0.11 & 0.46 \\
GBR & $d_{50}$ & 0.7 & 0.4 \\
GBR & $d_{90}$ & -0.05 & 0.24 \\
\hline Meandering & - & 0.8 & 0.3 \\
Graided & - & 0.9 & 0.4 \\
\hline GBD & $d_{50}$ & 0.5 & 0.2 \\
\hline \multicolumn{4}{c}{$\log _{10}(W / H)$} \\
\hline
\end{tabular}

$W_{*} \propto Q_{*}^{-0.1 \pm 0.02}$ and $H_{*} \propto Q_{*}^{-0.05 \pm 0.02}$ for the GBR threads. The width of the Bayanbulak threads shows the strongest correlation, yet even this correlation is mild. Finally, slopes are more strongly correlated with discharge than width and depth both for the GBR threads $\left(S_{*} \propto Q_{*}^{0.21 \pm 0.05}\right)$ and the Bayanbulak threads $\left(S_{*} \propto Q_{*}^{0.39 \pm 0.11}\right)$. However, most of the difference between the Bayanbulak and GBR threads is due to slopes well below the measurement precision. In all cases, the scatter is large, and all correlations fall within the standard deviation of the data set.

\section{Thread geometry}

We now analyze our rescaled measurements as a homogeneous statistical ensemble (Fig. 8). The means of the rescaled distributions of width, depth and slope all fall about 1 order of magnitude away from one, and their dispersion around this mean is also about 1 order of magnitude (Table 4). This observation supports the use of the threshold theory to scale the morphology of the Bayanbulak rivers. 

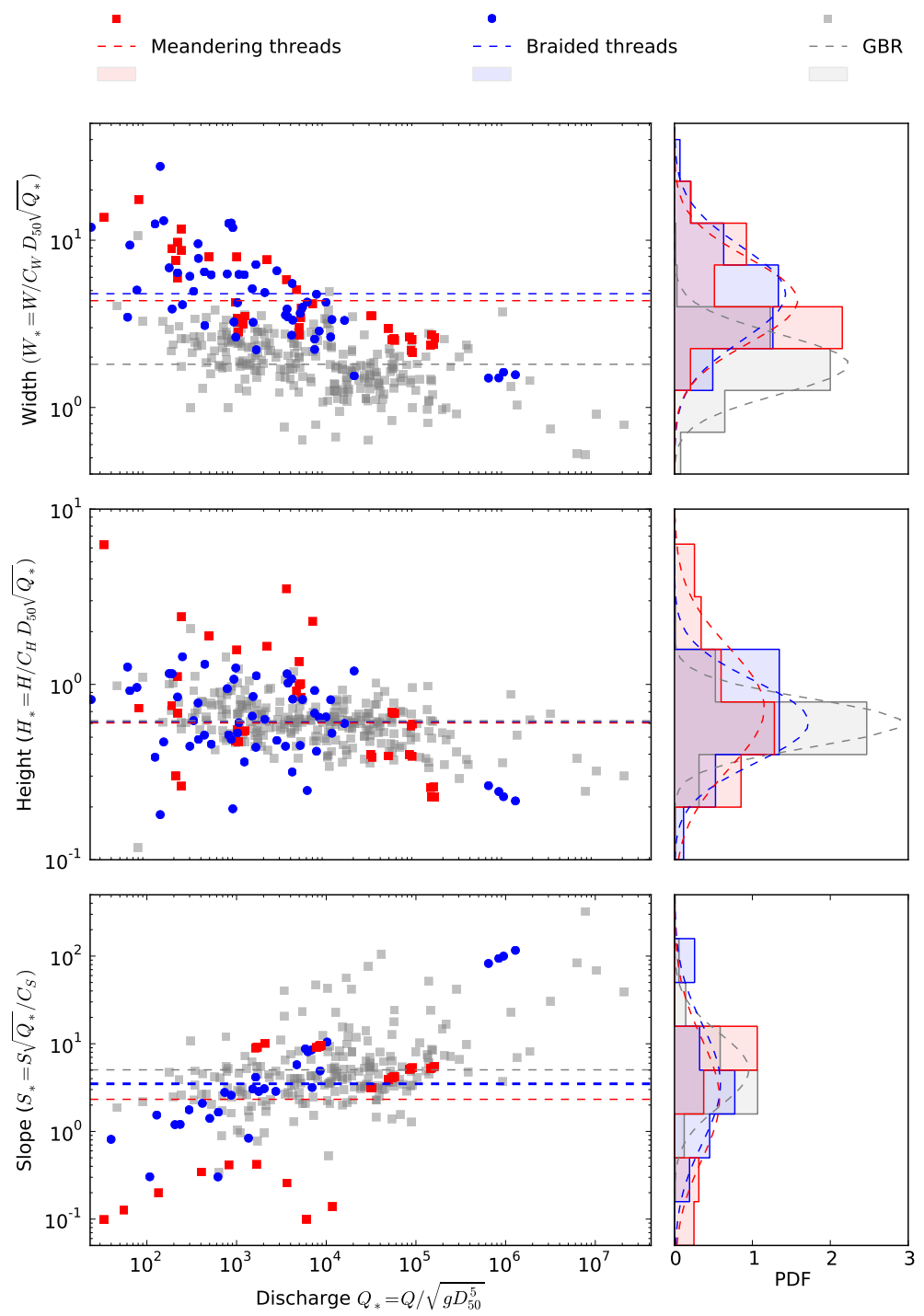

Figure 8. Left panels: rescaled width $\left(W_{*}\right)$, depth $\left(H_{*}\right)$ and slope $\left(S_{*}\right)$ as a function of rescaled water discharge $\left(Q_{*}\right)$. Rescaled quantities are from Eqs. (6) to (8). Threshold rivers correspond to rescaled width, depth and slope equal to 1. Right panels: probability density function of rescaled quantities, with fitted lognormal distributions.

The dispersion of the rescaled slope is more significant than that of width and depth. We believe that, in addition to the technical difficulties associated with the measurement of slope in the field (Sect. 3), the dispersion of the grain size explains this scatter. Indeed, gravels are broadly distributed in size, and unevenly distributed over the river bed (Guerit et al., 2014). Since the rescaling for slope involves the grain size $d_{\mathrm{S}}$ to the power of $5 / 4$, whereas this exponent is only $1 / 4$ for width and depth (Eqs. 6 to 8), we believe the grainsize dispersion impacts more strongly the rescaled slope than the rescaled width and depth.
The means of the distributions for braided threads and meandering threads differ by less than a factor of 2 , much smaller than the standard deviation. Fitting lognormal distributions to our data, we find that the meandering and braided threads from Bayanbulak cannot be distinguished from each other, at the $95 \%$ level of confidence. The depth and slope of the Bayanbulak threads are also not significantly different from the GBR threads. Finally, the width of the Bayanbulak threads is larger than that of the GBR ones. We therefore conclude that, within the natural variability of our observation, the sections of meandering and braided threads are geometrically similar although the morphology of the braided and meandering rivers looks qualitatively different (Figs. 2 and 4). Again, the use of $d_{90}$ instead of $d_{50}$ as a characteristic grain size does not alter this conclusion. 


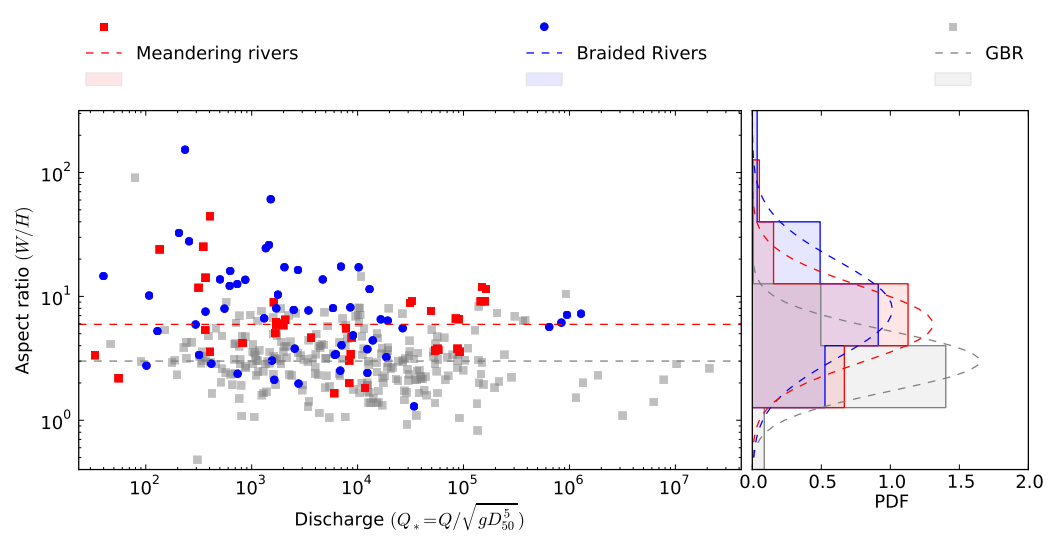

Figure 9. Aspect ratio of braided and meandering threads from Bayanbulak and the GBR data sets, as a function of rescaled water discharge $\left(Q_{*}\right)$.

According to the rescaling Eqs. (6) to (8) the aspect ratio of a river $W / H$ should be naturally detrended (Fig. 9). Indeed, the correlation coefficient of aspect ratio and discharge is less than 0.01 for all data sets. As expected, the aspect ratio of braided and meandering threads cannot be distinguished at the $95 \%$ level of confidence. Finally, the difference between the width of the Bayanbulak threads and that of the GBR threads also appears in the distribution of aspect ratios: the Bayanbulak aspect ratios are larger than the GBR ones.

\section{Conclusion}

Our measurements on gravel-bed rivers in the Bayanbulak Grassland reveal that braided threads are geometrically similar to meandering ones. Their size can be virtually detrended with respect to water discharge using the threshold theory. As a result, their aspect ratio is naturally detrended. These findings accord with recent observations in sand-bed rivers of the Kosi Megafan (Gaurav et al., 2015). They also accord with recent results from rivers of the Ganges-Brahmaputra plain (Gaurav, 2016).

The striking similarity between braided and meandering threads in gravel-bed and sand-bed rivers supports the view that fully developed braided rivers are essentially a collection of threads interacting with each other, rather than a single wide channel segmented by sediment bars. If confirmed, this would suggest that a braid results from the collective behavior of individual threads, the property and dynamics of which would be close to that of meandering threads (Sinha and Friend, 1994; Ashmore, 2013; Reitz et al., 2014).

Our observations, like those of Gaurav et al. (2015) or the GBR data set, are highly dispersed around their average value, which points at the influence of hidden parameters on their morphology. Among those, the intensity of sediment transport is likely to play a prominent role, at least in the case of the Bayanbulak rivers where both vegetation and grainsize distributions are relatively uniform over the grassland.
More specifically, field observations suggest that a heavier sediment load tends to increase the aspect ratio of a thread, other things being equal (Smith and Smith, 1984; Tal and Paola, 2010; Métivier and Barrier, 2012). This proposition remains speculative though, and needs to be thoroughly tested against dedicated field measurements, which we believe should include both braided and meandering threads. Finally, if the sediment discharge is indeed the most prominent parameter after water discharge, its influence on the geometry of a channel should also manifest itself in laboratory experiments.

Acknowledgements. This paper is dedicated to the dear memory of Baisheng Ye. The Bayanbulak field campaign was the last we carried out together, before he accidentally died on assignment in Tibet. This work is under the aegis of the SALADYN international associated laboratory. It is IPGP contribution 3685.

Edited by: D. Parsons

\section{References}

Andrews, E. D.: Bed-material entrainment and hydraulic geometry of gravel-bed rivers in Colorado, Geol. Soc. Am. Bull., 95, 371378, 1984.

Ashmore, P. E.: How do gravel-bed rivers braid?, Can. J. Earth Sci., 28, 326-341, 1991.

Ashmore, P. E.: Fluvial Geomorphology, in: vol. 9 of Treatise on Geomorphology, chapter Morphology and dynamics of braided rivers, Academic Press, San Diego, CA, 289-312, 2013.

Brauderick, C. A., Dietrich, W. E., Leverich, G. T., and Sklar, L. S.: Experimental evidence for the conditions necessary to sustain meandering in coarse-bedded rivers, P. Natl. Acad. Sci., 1693616941, doi:10.1073/pnas.0909417106, 2009.

Bunte, K. and Abt, S.: Sampling surface and subsurface particlesize distributions in wadable gravel- and cobble-bed streams for analyses in sediment transport, hydraulics, and streambed monitoring, Technical report, United States Department of Agriculture, Forest Service, Fort Colins (CO), 451pp., 2001. 
Church, M.: Pattern of instability in a wandering gravel bed channel, in: Modern and ancient fluvial systems, Special Publication, Int. Assoc. Sedimentol., 6, 169-180, 1983.

Church, M. and Gilbert, R.: Glaciofluvial and glaciolacustrine sedimentation, in: vol. 23 of Special Publication, chapter Proglacial Fluvial and Lacustrine Environments, SEPM, 22-100, 1975.

Church, M. and Rood, K.: Catalogue of Alluvial River Channel Regime Data, Univ. British Columbia, Department of Geography, Vancouver, 110 pp., 1983.

Devauchelle, O., Josserand, C., Lagrée, P., and Zaleski, S.: Morphodynamic modeling of erodible laminar channels, Phys. Rev. E, 76, 056318, doi:10.1103/PhysRevE.76.056318, 2007.

Dijk, W., Lageweg, W., and Kleinhans, M.: Experimental meandering river with chute cutoffs, J. Geophys. Res.-Earth, 117, F03023, doi:10.1029/2011JF002314, 2012.

Ferguson, R.: Hydraulic and sedimentary controls of channel pattern, in: River channels: environment and process, Blackwell, Oxford, UK, 129-158, 1987.

Fredsøe, J.: Meandering and braiding of rivers, J. Fluid Mech., 84, 609-624, 1978.

Fujita, Y. and Muramoto, Y.: Studies on the process of development of alternate bars, Bulletin of the Disaster Prevention Research Institute, Kyoto University, 35, 3, 55-86, 1985.

Gaurav, K.: Morphology of alluvial rivers, $\mathrm{PhD}$ thesis, IPGP, Paris, France, 135 pp., 2016.

Gaurav, K., Métivier, F., Devauchelle, O., Sinha, R., Chauvet, H., Houssais, M., and Bouquerel, H.: Morphology of the Kosi megafan channels, Earth Surf. Dynam., 3, 321-331, doi:10.5194/esurf-3-321-2015, 2015.

Glover, R. E. and Florey, Q.: Stable channel profiles, US Department of the Interior, Bureau of Reclamation, Design and Construction Division, Denver, CO, 42 pp., 1951.

Gran, K. and Paola, C.: Riparian vegetation controls on braided stream dynamics, Water Resour. Res., 37, 3275-3283, 2001.

Guerit, L., Barrier, L., Narteau, C., Métivier, F., Liu, Y., Lajeunesse, E., Gayer, E., Meunier, P., Malverti, L., and Ye, B.: The Grainsize Patchiness of Braided Gravel-Bed Streams - example of the Urumqi River (northeast Tian Shan, China), Adv. Geosci., 37, 27-39, doi:10.5194/adgeo-37-27-2014, 2014.

Henderson, F. M.: Stability of alluvial channels, T. Am. Soc. Civ. Eng., 128, 657-686, 1963.

King, J. G., Emmett, W. W., Whiting, P. J., Kenworthy, R. P., and Barry, J. J.: Sediment transport data and related information for selected coarse-bed streams and rivers in Idaho, Gen. Tech. Rep. RMRS-GTR-131, Department of Agriculture, Forest Service, Rocky Mountain Research Station, Fort Collins, CO, p. 26, 2004.

Kleinhans, M. G. and van den Berg, J. H.: River channel and bar patterns explained and predicted by an empirical and a physicsbased method, Earth Surf. Proc. Land., 36, 721-738, 2011.

Leopold, L. B. and Wolman, M. G.: River Channel Patterns, Technical Report 282-B, U. S. Geol. Survey, Washington, 283-300, 1957.

Métivier, F. and Barrier, L.: Alluvial landscape evolution: what do we know about metamorphosis of gravel bed meandering and braided streams, in: Gravel-bed Rivers: processes, tools, environments, chapter 34, edited by: Church, M., Biron, P., and Roy, A., Wiley \& Sons, Chichester, 474-501, 2012.
Mosley, M.: Response of braided rivers to changing discharge, J. Hydrol. New Zeal., 22, 18-67, 1983.

Parker, G.: On the cause and characteristic scales of meandering and braiding in rivers, J. Fluid Mech., 76, 457-480, 1976.

Parker, G.: Transport of gravel and sand mixtures, in: Sedimentation engineering: processes, management, modeling, and practice, vol. 110, chapter 3, edited by: Garcia, M. H., Am. Soc. Civ. Eng., Reston, VA, 165-251, 2008.

Parker, G., Wilcock, P., Paola, C., Dietrich, W. E., and Pitlick, J.: Quasi-universal relations for bankfull hydraulic geometry of single-thread gravel-bed rivers, J. Geophys. Res.-Earth, 112, 121, 2007.

Reitz, M. D., Jerolmack, D. J., Lajeunesse, E., Limare, A., Devauchelle, O., and Métivier, F.: Diffusive evolution of experimental braided rivers, Phys. Rev. E, 89, 052809, doi:10.1103/PhysRevE.89.052809, 2014.

Sanders, L.: A manual of field hydrogeology, Prentice-Hall, Inc., Englewood Cliffs, NJ, USA, p. 381, 1998.

Scherrer, B.: Biostatistique, G. Morin, Chicoutimi, Québec, p. 850, 1984.

Schumm, S. A.: River variability and complexity, Cambridge Univ Pr., Cambridge, 236p., 2005.

Seizilles, G.: Forme d'équilibre d'une rivière, $\mathrm{PhD}$ thesis, Université Paris Diderot, Paris, p. 179, 2013.

Seizilles, G., Devauchelle, O., Lajeunesse, E., and Métivier, F.: Width of laminar laboratory rivers, Phys. Rev. E, 87, 052204, doi:10.1103/PhysRevE.87.052204, 2013.

Sinha, R. and Friend, P.: River systems and their sediment flux, indo-gangetic plains, northern bihar, india, Sedimentology, 41, 825-845, 1994.

Smith, N. D. and Smith, D. G.: William River: An outstanding example of channel widening and braiding caused by bed-load addition, Geology, 12, 78, 1984

Sokal, R. and Rohlf, F.: Biometry, W. H. Freeman, p. 850, 1995.

Tal, M. and Paola, C.: Dynamic single-thread channels maintained by the interaction of flow and vegetation, Geology, 35, 347-350, 2007.

Tal, M. and Paola, C.: Effects of vegetation on channel morphodynamics: results and insights from laboratory experiments, Earth Surf. Proc. Land., 35, 1014-1028, 2010.

Wolman, M. G.: A method for sampling coarse river bed material, EOS Trans. AGU, 36, 655-663, 1954.

Yalin, M. S. and Ferreira da Silva, A. M.: Fluvial Processes, International Association of Hydraulic Engineering and Research Monograph, Delft, p. 197, 2001.

Yang, Q. and Cui, C.: Impact of climate change on the surface water of kaidu river basin, J. Geogr. Sci., 15, 20-28, 2005.

Zhang, B., Yao, Y.-H., Cheng, W.-M., Zhou, C., Lu, Z., Chen, X., Alshir, K., ErDowlet, I., Zhang, L., and Shi, Q.: Human-induced changes to biodiversity and alpine pastureland in the bayanbulak region of the east tienshan mountains, Mount. Res. Dev., 22, 383-389, 2002.

Zhang, Y., Li, B., Bao, A., Zhou, C., Chen, X., and Zhang, X.: Study on snowmelt runoff simulation in the kaidu river basin, Sci. China Ser. D, 50, 26-35, 2007.

Zolezzi, G., Bertoldi, W., and Tubino, M.: Morphodynamics of bars in gravel-bed rivers: Bridging analytical models and field observations, in: Gravel-Bed Rivers: Processes, Tools, Environments, Wiley \& Sons, Chichester, 69-89, 2012. 